\title{
The Optimization Technique for Joint Discrete Search and Detection of Observation Objects
}

\author{
Hennadii Khudov', Irina Khizhnyak ${ }^{2}$, Iryna Yuzova ${ }^{3}$, Oleksii Baranik ${ }^{4}$, Galina Semiv ${ }^{5}$, Semen \\ Bondarenko ${ }^{6}$, Olexander Tytarenko ${ }^{7}$ \\ ${ }^{1}$ Department of Radar Troops Tactic, Ivan Kozhedub Kharkiv National Air Force University, Kharkiv, Ukraine, \\ 2345kh_hg@ukr.net \\ ${ }^{2}$ Department of Mathematical and Software Automated Control Systems, Ivan Kozhedub Kharkiv National Air \\ Force University, Kharkiv, Ukraine, khizh_ia@ukr.net \\ ${ }^{3}$ Department of Reserve Officers Training, Ivan Kozhedub Kharkiv National Air Force University, Kharkiv, \\ Ukraine,uzik25@ukr.net \\ ${ }^{4}$ Department of Aviation Armament Complexes, Ivan Kozhedub Kharkiv National Air Force University, Kharkiv, \\ Ukraine, kozaktur@i.ua \\ ${ }^{5}$ Department of Rocket Artillery Armament, Hetman Petro Sahaidachnyi National Army Academy, Lviv, \\ Ukraine, galinasemiv1985@gmail.com \\ ${ }^{6}$ Department of Rocket Artillery Armament, Hetman Petro Sahaidachnyi National Army Academy, Lviv, \\ Ukraine, bondarenko_semen2@ukr.net \\ ${ }^{7}$ Department of Air Forces, Ivan Chernyakhovsky National Defense University of Ukraine, Kyiv, Ukraine, \\ tabzrv@ukr.net
}

\begin{abstract}
Briefly analyzes the main results of solving problems of search and detection of objects of observation in information systems. A discrete search zone is introduced. The task is to find the optimal Bayes decision rule in the current discrete search zone. The Bayesian decision-making rule is clarified when jointly optimizing discrete search and detection of observation objects.
\end{abstract}

Key words : information system, joint search and detection of objects, discrete search and detection of objects, the time of searching and finding objects, Bayes criterion of minimum average risk.

\section{INTRODUCTION}

Today, a whole class of information systems is used to solve the problem of searching and detecting objects in conditions of limited search potential [1-7]. This article assesses the possibility of reducing the requirements for search potential due to the joint Bayes optimization of the search and detection of objects.

The goal of research is to reduce the time of searching and finding objects by optimizing the spatio-temporal distribution of the search potential. It takes into account the specifics of the operation of the information system in discrete search mode.

\subsection{Problem analysis}

The tasks of searching and detecting objects in information systems are currently being solved independently of each other. This applies to both radar $[1,3,4-8,10]$ and optoelectronic [2, 9, 11-16] systems. There are optimization methods that consider search and detection as a single task only in the production plan. Solutions are obtained for the individual components of this task.

In [3], the problem of joint search and detection optimization was solved as follows. For the case of a continuous a certain area of survey $\Omega$, an improved Bayes rule for deciding on the detection of an object is formulated. This rule is as follows. When solving the task of testing a simple hypothesis against a simple alternative, the joint optimization of the search and detection of objects reduces to finding a uniformly optimal search strategy, calculating the maximum of the unconditional likelihood ratio in the current area of view and comparing it with the threshold.

In [17], the results of joint search and detection of objects efficiency are briefly analyzed. The weight criterion of an optimality of detection in a zone of search elementary cell is formulated. The differential characteristics of Bayes criterion of a minimum of average risk are taken into account. The weight criterion of joint optimization of search and detection of objects in the current zone of search is specified. Expression for the ratio of likelihood in the current zone of search is received. 
Hennadii Khudov et al., International Journal of Emerging Trends in Engineering Research, 8(2), February 2020, $533-538$

Consider the results obtained in $[3,17,18]$ for the case of solving task of the discrete searching and detecting a stationary single object.

\section{MAIN MATERIAL}

Consider the task of joint Bayesian optimization of discrete search and detection of objects. For this will use the criterion of minimum average risk, namely, the average value of the decision-making fee when testing statistical hypotheses.

For further research, divide the area of search $\Omega$ into $N$ sub-areas $\Omega_{i}$, such that $\sum_{i=1}^{N} \Omega_{i}=\Omega$. The average risk in sub-area $\Omega_{j}$ is denoted as $R_{j}$.

Introduce the current area of search $\Omega\left(t_{i}\right)=\sum_{j\left(t_{i}\right)} \Omega_{j}$,

where $j$ - number of sub-area of search and detection of an object at time $t$. Index $j$ indicates the serial number of the sub-area in which the search and detection of the object at a time $t_{i}$.

Set the task of finding the optimal Bayes decision rule in the current discrete area of search $\Omega\left(t_{i}\right)$. With this formulation of the task, an additional optimization parameter appears, namely, the current dimensions and position of the discrete area $\Omega\left(t_{i}\right)$ in the common area $\Omega$.

Therefore, conditions are created for finding the optimal (by the Bayes criterion of minimum average risk) strategy for joint search and detection of an object in the search area of a discrete structure. A solution to such a problem, both in search theory and in detection theory, was previously absent

According to $[3,17,18]$, the average risk in the current area of search $\Omega\left(t_{i}\right)$ now can found as:

$$
\begin{aligned}
& R\left(t_{i}\right)=\sum_{\Omega\left(t_{i}\right)} R_{j}=R_{0}-\left(\left(I_{10}-I_{11}\right)\right. \\
& \left.\sum_{\Omega\left(t_{i}\right)} P_{1 j}\left(\gamma_{1}, t_{i}\right)-\left(I_{01}-I_{00}\right) \sum_{\Omega\left(t_{i}\right)} P_{0 j}\left(\gamma_{1}, t_{i}\right)\right),
\end{aligned}
$$

where $P_{1 j}\left(\gamma_{1}, t_{i}\right)$ - the current value of the unconditional probability of the correct detection of objects in the $j$-th sub-area at a time $t$;

$P_{0 j}\left(\gamma_{1}, t_{i}\right)$ - the current value of the unconditional probability of false alarm in the $j$-th sub-area at a time $t$.
Assume that $R_{0}$ is a non-negative constant for the current area of search $\Omega\left(t_{i}\right)$ at time $t$. Write the Bayes rule for testing simple hypothesis $H_{0}$ against simple alternative $H_{1}$ in the current discrete sub-area $\Omega\left(t_{i}\right)$ of area $\Omega$ as follows:

$$
\frac{\sum_{\Omega\left(t_{i}\right)} P_{1 j}\left(\gamma_{1}, t_{i}\right)}{\sum_{\Omega\left(t_{i}\right)} P_{0 j}\left(\gamma_{1}, t_{i}\right)} \stackrel{\substack{\gamma_{1} \\>}}{>} \frac{I_{01}-I_{00}}{I_{10}-I_{11}},
$$

Passing to the unconditional likelihood ratio

$$
l\left(t_{i}\right)=\frac{\sum_{\Omega\left(t_{i}\right)} P_{1 j}\left(\gamma_{1}, t_{i}\right)}{\sum_{\Omega\left(t_{i}\right)} P_{0 j}\left(\gamma_{1}, t_{i}\right)},
$$

write expression (2) as follows:

$$
l\left(t_{i}\right)_{\gamma_{0}}^{\gamma_{1}} \frac{I_{01}-I_{00}}{I_{10}-I_{11}},
$$

Thus, the optimal Bayes rule (3) for testing a simple hypothesis against a simple alternative, which was obtained on the basis of expressions (1) and (2), is to maximize the unconditional likelihood ratio $l\left(t_{i}\right)$ in the current discrete area $\Omega\left(t_{i}\right)$ and compare it with the threshold (4):

$$
c_{b}=\frac{I_{01}-I_{00}}{I_{10}-I_{11}},
$$

At that,

if $l(t) \geq c_{b}$, then decision $\gamma_{1}$ is made, that is, the hypothesis $H_{0}$ is rejected;

if $l(t)<c_{b}$, then decision $\gamma_{0}$ is made, that is, hypothesis $H_{0}$ is accepted.

In accordance with (2) optimization should be carried out by: parameters of conditional probability of correct detection of $P\left(\gamma_{1} / H_{1}, x\right)$ in sub-areas $\Omega_{j}$;

parameters of the current discrete area of search $\Omega\left(t_{i}\right)$.

Consider an important special case. Assume that, similarly to the Neumann-Pearson criterion, the value of the unconditional probability of false alarm in sub-area $\Omega_{j}$ at a time $t$ is fixed at a constant level $-P_{0 j}\left(\gamma_{1}, t_{i}\right)$. Then, 
according to expression (2), finding the maximum of the unconditional likelihood ratio reduces to finding the maximum of the unconditional probability of the correct detection of the object in the current discrete sub-area $\Omega\left(t_{i}\right)$.

Thus, to find the optimal Bayes decision rule in the current discrete area $\Omega\left(t_{i}\right)$ of the common area $\Omega$, along with the solution of the hypothesis testing problem in this area, the problem of finding the optimal object search strategy (using the Bayes criterion of minimum average risk) must be solved.

Search strategy $\lambda\left(\Omega_{j}, t_{i}\right)$ is a rule that at any moment of time $t_{\lambda}$ establishes in which sub-area $\Omega_{j}$ of the area the search should be carried out and with what energy costs.

For further research, introduce the main restrictions on the search strategy, which are usually used in search theory. Namely, require that search strategy $\lambda\left(\Omega_{j}, t_{i}\right)$ be Ttruncated, that is, $\lambda\left(\Omega_{j}, t_{i}\right)=0$ for $t_{i}>T$ and $x \in \Omega$. That is, the condition of compulsory viewing of area $\Omega$ during the search $T$ must be fulfilled.

It's obvious that:

$$
\begin{aligned}
& \lambda\left(\Omega_{j}, t_{i}\right)>0, \quad \text { for } \quad \Omega_{j} \in \Omega\left(t_{i}\right) ; \\
& \lambda\left(\tilde{\Omega}, t_{i}\right)=0, \quad \text { for } \quad \tilde{\Omega} \in \Omega / \Omega\left(t_{i}\right) .
\end{aligned}
$$

Assume that the search strategy should be constant for all sub-areas that are viewed at a fixed moment of time $t_{i}$. The measure of the current area $\Omega\left(t_{i}\right)$ consists of the sub-areas $\Omega_{j}$ viewed at the moment $t_{i}$.

$$
\Omega\left(t_{i}\right)=\sum_{j} \Omega_{j}
$$

In addition to the above properties of the search strategy, require that it satisfy the optimality condition. This condition is that if each T-truncated strategy $\lambda\left(\Omega_{j}, t_{i}\right)$ has a functional $P\left(\lambda\left(\Omega_{j}, t_{i}\right)\right)$, then strategy $\lambda_{\text {opt }}\left(\Omega_{j}, t_{i}\right)$ will be optimal if:

$$
P\left(\lambda_{\text {opt }}\left(\Omega_{j}, t_{i}\right)\right)=\sup P\left(\lambda\left(\Omega_{j}, t_{i}\right)\right),
$$

where $P\left(\lambda\left(\Omega_{j}, t_{i}\right)\right)$ - unconditional probability of correct detection of an object at time $t_{i}$ at strategy $\lambda\left(\Omega_{j}, t_{i}\right)$.

Also require that the search strategy be optimal for any moment of time $T$ of the end of the search. That is, at whatever moment of time the search would not be interrupted, up to this moment of time it should be optimal according to the criterion of the maximum unconditional probability of correct detection.

From the analysis of the results for the selection of search strategies that are studied in the theory of search, of all the strategies, the class of uniformly optimal search strategies most fully satisfies expressions (5)-(7).

Strategy $\lambda\left(\Omega_{j}, t_{i}\right)$ is uniformly optimal if any T-truncated strategy is optimal, i.e.

$$
P\left(\lambda\left(\Omega_{j}, t_{i}\right)\right)=P\left(\lambda_{\text {opt }}\left(\Omega_{j}, t_{i}\right)\right), \quad \forall t_{i} \leq T .
$$

Thus, when solving the problem of finding, according to the Bayes criterion of the minimum of the average risk of the search strategy and object detection, the uniformly optimal search strategy is optimal. In accordance with which the current sizes and position of sub-area $\Omega\left(t_{i}\right)$ in the common search area $\Omega$ should be selected.

In accordance with expression (2) with a value of the unconditional probability of false alarm fixed at a constant level, the optimization task is formulated as follows:

$$
\begin{gathered}
P_{1}\left(\gamma_{1}, t_{i}\right) \rightarrow \max \\
\lambda\left(\Omega_{j}, t_{i}\right) \geq 0, \quad t_{i}>0 \\
\sum_{\Omega\left(t_{i}\right)} \lambda\left(\Omega_{j}, t_{i}\right)=L_{0}, \quad t_{i}>0 ; \\
\sum_{j} \lambda\left(\Omega_{j}, t_{i}\right)=\varphi\left(t_{i}\right) \\
\sum_{\Omega\left(t_{i}\right)} \varphi\left(t_{i}\right)=L_{0} t_{i},
\end{gathered}
$$

where $P_{1}\left(\gamma_{1}, t_{i}\right)$ - the unconditional probability of detecting an object at time $t_{i}$ in the discrete area of search $\Omega\left(t_{i}\right)$;

$L_{0}-$ characterizes the power of a search engine;

$\varphi\left(t_{i}\right)$ - search effort in search area $\Omega$ at time $t_{i}$.

It is rather difficult to obtain a solution to the optimization task (9) for the discrete case. Therefore, we reformulate the optimization task (9) somewhat and use the well-known approach to solve it.

The main variable will be considered as $0 \leq P_{1 j} \leq 1-$ the conditional probability that the object will be detected when viewing the sub-area with the number $l$ of area $\Omega_{j}$. But 
provided that the object is actually located in area $\Omega_{j}$ and has not been previously detected. It is understood that scans with numbers $1,2, \ldots, l-1$ of sub-area $\Omega_{j}$ were carried out earlier, and not necessarily sequentially, one after another.

Introduce the notation $q_{l j}=1-P_{l j}$. Let area $\Omega_{j}$ be viewed $n_{j}$ times. Then the conditional probability that an object in area $\Omega_{j}$ will be detected when viewed with number $l$ is $P_{i j} \prod_{i=1}^{l-1} q_{i j}$.

If all areas $\Omega_{j}, \quad j=\overline{1, N}$, are viewed, and area $\Omega_{j}$ is viewed $n_{j}$ times, then there is an unconditional probability of detecting an object during this sequence of views:

$$
\sum_{j=1}^{N} P_{j} \sum_{l=1}^{n_{j}} P_{i j} \prod_{i=1}^{l-1} q_{i j}
$$

where $P_{j}$ - a priori probability of the distribution of the location of the object in area $\Omega_{j}$.

If condition $\sum_{j=1}^{N} n_{j} S_{j} \leq C$ is introduced,

where $C$ - total search potential allocated at time $T$;

$n_{j} \in Z=\{0,1,2, \ldots\}$

$S_{j}$ - area of the $j$-st sub-area of the search area $\Omega$,

then obtain the following optimization task:

$$
\begin{gathered}
\sum_{j=1}^{N} P_{j} \sum_{l=1}^{n_{j}} P_{i j} \prod_{i=1}^{l-1} q_{i j} \rightarrow \max ; \\
\sum_{j=1}^{N} n_{j} S_{j} \leq C ; \\
n_{j} \in Z, \quad j=\overline{1, N} .
\end{gathered}
$$

In the optimization task (11), there are two types of unknown quantities:

search efforts expressed in whole numbers $n_{1}, n_{2}, \ldots, n_{k}$;

areas $\Omega_{1}, \Omega_{2}, \ldots, \Omega_{N}$.
Rewrite the optimization task (11) in the form of a dynamic programming task:

$$
\begin{aligned}
& f_{N}(\mathrm{z})=\max _{n_{j}, j=1, N}\left\{\sum_{j=1}^{N} P_{j} \sum_{l=1}^{n_{j}} P_{i j} \prod_{i=1}^{l-1} q_{i j}\right\}, \\
& \text { where } \quad \sum_{j=1}^{N} n_{j} S_{j} \leq z .
\end{aligned}
$$

Solving (12) using the Bellman optimization theory, obtain the optimal number of views of each area. After finding the optimal number of views of each area, determine the viewing order of these areas.

At the same time, set the condition for ensuring the minimum time for searching and detecting an object. For this we use the relationship:

$$
\frac{P_{j} P\left(\gamma_{1} / H_{1}, i, j\right) \prod_{l=1}^{i-1} \bar{P}\left(\gamma_{1} / H_{1}, l, j\right)}{S_{j}},
$$

where $P\left(\gamma_{1} / H_{1}, i, j\right)=1-\bar{P}\left(\gamma_{1} / H_{1}, i, j\right)$;

$i$ - number of views of the $j$-st area, $i=\overline{1, u_{i-1}^{o p t}}$;

$j=\overline{1, k}, k-$ total number of areas in the search area $\Omega$.

Having ordered relations (13) in descending order, it is necessary to solve the search and detection task in the same order.

Based on the studies performed, the following refined optimal Bayes decision rule can be formulated. When solving the task of testing a simple hypothesis against a simple alternative, the joint optimization of discrete search and object detection is reduced to:

finding a uniformly optimal search strategy;

calculating the maximum unconditional likelihood ratio in the current discrete area $\Omega\left(t_{i}\right)$;

comparing it with a threshold.

When this rule is fulfilled, the mean time until the object is detected can be calculated by (14):

$$
T_{m}=\int_{0}^{T} Q(t) d t
$$


Hennadii Khudov et al., International Journal of Emerging Trends in Engineering Research, 8(2), February 2020, $533-538$

where $Q(t)$ - unconditional probability of non-detection of an object.

Taking into account that $Q(t)=1-P_{1}\left(\gamma_{1}, t\right)$ for discrete search and detection, write expression (15):

$$
T_{m}=\sum_{j}\left(1-P_{j} P_{1}\left(t_{j}\right)\right) \Delta t_{j},
$$

where $P_{1}\left(t_{j}\right)$ - conditional probability of detecting an object in a sub-area $\Omega_{j}$;

$\Delta t_{j}$ - the time allotted to view a sub-area $\Omega_{j} ;$

$\sum_{j} \Delta t_{j}=T$

For quantitative estimates, assume that $P_{j}=P_{1}(t), \Delta t_{j}=\Delta t$. Consider two special cases:

I. $P_{1}=P_{2}=0,5 ; \Delta t=0,5 T$

II. $P_{1}=0,7 ; P_{2}=0,3 ; \Delta t=0,5 T$.

For these cases get:

I. $T_{m} \approx 0,75 T$;

II. $T_{m} \approx 0,71 T$.

Can conclude that with the joint optimization of search and detection, a gain in average time is provided before the detection of an object

\section{CONCLUSION}

Thus, the joint optimization of discrete search and detection of objects can reduce time and, accordingly, reduce the energy cost of detecting objects.

Introduced the current discrete area of view. The task of finding the optimal Bayes decision-making rule in the introduced current discrete area of view is posed and solved.

The specified Bayes optimal decision rule is formulated. When solving the task of testing a simple hypothesis against a simple alternative, the joint optimization of discrete search and object detection is reduced to:

finding a uniformly optimal strategy for finding an object in discrete cells of the search area;

calculating the maximum unconditional likelihood ratio in the current group of search subdomains;

comparing it with a threshold.

\section{REFERENCES}

1. R. Hoseinnezhad, B.-N. Vo, and B.-T. Vo, Visual Tracking in Background Subtracted Image Sequences via Multi-Bernoulli Filtering, IEEE Trans. Signal Processing, Vol. 61, No. 2, 2013, pp. 392-397.

2. I. Ruban, H. Khudov, O. Makoveichuk, I. Khizhnyak, V. Khudov, V. Podlipaiev, V. Shumeiko, O. Atrasevych, A. Nikitin, and R. Khudov. Segmentation of opticalelectronic images from on-board systems of remote sensing of the Earth by the artificial bee colony method, Eastern-European Journal of Enterprise Technologies, № 2/9 (98), 2019, pp. 37-45. DOI: https://doi.org/10.15587/1729-4061.2019.161860.

3. H. Khudov, I. Khizhnyak, F. Zots, G. Misiyuk, and O. Serdiuk. The Bayes Rule of Decision Making in Joint Optimization of Search and Detection of Objects in Technical Systems, IJETER, № 8(1), 2020, pp. 7-12. DOI: https://doi.org/10.30534/ijeter/2020/02812020.

4. V. Lishchenko, V. Chaliy, G. Khudov, and A. Zvonko. Proposals for Improving of Air Surveillance Informativity in MIMO Radar Systems Based on Two-Dimensional Radars, in Intern. Scient.-Pract. Conf. Problems of Infocommunications. Science and Technology (PIC S\&T), 2018, pp. 153-156. DOI: https://doi.org/10.1109/infocommst.2018.8632052.

5. M. Iasechko, M. Mozhaiev, I. Manzhai, M. Volk, V. Manoylo, O. Ochkurenko, D. Maksuita, V. Larin, A. Markov, and O. Kostyria. Conditions for Reliable Transmission of Information Over Long Distances Using a Powerful Electromagnetic Radiation, IJATCSE. $\quad 8(1), \quad 2020, \quad$ pp. 138-144. DOI: https://doi.org/10.30534/ijeter/2020/18812020.

6. H. Khudov, A. Fedorov, D. Holovniak, and G. Misiyuk. Improving the Efficiency of Radar Control of Airspace with the Multilateration System Use, in Intern. Scient.-Pract. Conf. Problems of Infocommunications. Science and Technology (PIC S\&T), 2018, pp. 680-684. DOI: https://doi.org/10.1109/infocommst.2018.8632141.

7. O. Turinskyi, M. Iasechko, V. Larin, D. Dulenko, V. Kravchenko, O. Golubenko, D. Sorokin, and O. Zolotuin. Model and development of plasma technology for the protection of radio-electronic means of laser emission, IJATCSE. 8(5), 2019, pp. 2429-2433. doi:10.30534/IJATCSE/2019/85852019.

8. V. Lishchenko, H. Khudov, V. Tiutiunnyk, V._Kuprii, F.ZZots, and G._Misiyuk. The Method of Increasing the Detection Range of Unmanned Aerial Vehicles In Multiradar Systems Based on Surveillance Radars, in 2019 IEEE 39th International Conference on Electronics and Nanotechnology (ELNANO), 2019. DOI: https://doi.org/10.1109/ELNANO.2019.8783263.

9. H. Khudov, A. Zvonko, S. Kovalevskyi, V. Lishchenko, F. Zots. Method for the detection of smallsized air objects by observational radars, Eastern-European Journal of Enterprise Technologies, № 2/9 (92), 2018, pp. 
Hennadii Khudov et al., International Journal of Emerging Trends in Engineering Research, 8(2), February 2020, $533-538$

$61-68$.

DOI:https://doi.org/10.15587/1729-4061.2018.126509.

10. M. Iasechko, Y. Gnusov， I. Manzhai， O. Uhrovetskyi, V. Manoylo, A. Iesipov, O. Zaitsev, M. Volk, and O. Volk. Determination of Requirements for the Protection of Radio-Electronic Equipment from the Terroristic Influence by Electromagnetic Radiation, IJATCSE. 7(12), 2019, pp. 772-777. DOI: https://doi.org/10.30534/ijeter/2019/077122019.

11. H. Khudov, I. Ruban, O. Makoveichuk, H. Pevtsov, V. Khudov, I. Khizhnyak, S. Fryz, V. Podlipaiev, Y. Polonskyi, and R. Khudov. Development of methods for determining the contours of objects for a complex structured color image based on the ant colony optimization algorithm, Eureka: Physics and Engineering, № 1, 2020, pp. 34-47. DOI: https://doi.org/10.21303/2461-4262.2020.001108.

12. I. Ruban, V. Khudov, O. Makoveichuk, H. Khudov, and I. Khizhnyak. A Swarm Method for Segmentation of Images Obtained from On-Board Optoelectronic Surveillance Systems, in Intern. Scient.-Pract. Conf. Problems of Infocommunications. Science and Technology (PIC S\&T), 2018, pp. 613-618. DOI: https://doi.org/10.1109/infocommst.2018.8632045.

13. I. Ruban, H. Khudov, V. Khudov, I. Khizhnyak, and O. Makoveichuk. Segmentation of the images obtained from onboard optoelectronic surveillance systems by the evolutionary method, Eastern-European Journal of Enterprise Technologies, № 5/9 (89), 2017, pp. 49-57. DOI: https://doi.org/10.15587/1729-4061.2017.109904.

14.I. Ruban, H. Khudov, O. Makoveichuk, M. Chomik, V. Khudov, I. Khizhnyak, V. Podlipaiev, Y. Sheviakov, O. Baranik, and A. Irkha. Construction of methods for determining the contours of objects on tonal aerospace images based on the ant algorithms, Eastern-European Journal of Enterprise Technologies, № 5/9 (101), 2019, pp. 25-34.

https://doi.org/10.15587/1729-4061.2019.177817.

15. I. Ruban, H. Khudov, O. Makoveichuk, I. Khizhnyak, N. Lukova-Chuiko, G. Pevtsov, Y. Sheviakov, I. Yuzova, Y. Drob, and O. Tytarenko, Method for determining elements of urban infrastructure objects based on the results from air monitoring, Eastern-European Journal of Enterprise Technologies, № 4/9 (100), 2019, pp. 52-61.

DOI: https://doi.org/10.15587/1729-4061.2019.174576.

16. I. Ruban, V. Khudov, H. Khudov, and I. Khizhnyak. An Improved Method for Segmentation of a Multiscale Sequence of Optoelectronic Images, in Intern. Scient.-Pract. Conf. Problems of Infocommunications. Science and Technology (PIC S\&T), 2017, pp. 137-140. DOI: https://doi.org/10.1109/INFOCOMMST.2017.8246 367.

17. G. V. Khudov, Features of optimization of two-alternative decisions by joint search and detection of objects. Problemy Upravleniya I Informatiki (Avtomatika), 2003, № 5, pp. 51-59.
18. T. D. Wickens. Elementary signal detection theory, New York, NY: Oxford University Press, 2002, 277 p. https://doi.org/10.1093/acprof:oso/9780195092509.001.0001 